The Journal of Laryngology \& Otology

http://journals.cambridge.org/JLO

Additional services for The Journal of Laryngology \& Otology:

Email alerts: $\underline{\text { Click here }}$

Subscriptions: $\underline{\text { Click here }}$

Commercial reprints: Click here

Terms of use : $\underline{\text { Click here }}$

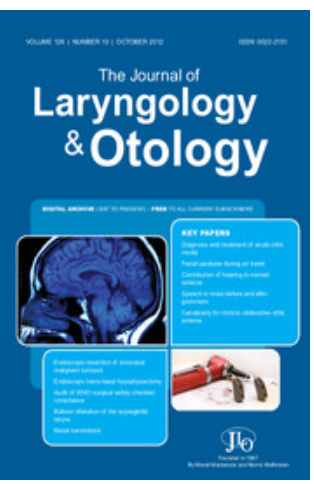

\title{
CT and MR imaging in lateral sinus thrombosis
}

R. M. Irving, N. S. Jones, M. A. Hall-Craggs and B. Kendall

The Journal of Laryngology \& Otology / Volume 105 / Issue 08 / August 1991, pp 693 - 695

DOI: 10.1017/S0022215100117050, Published online: 29 June 2007

Link to this article: http://journals.cambridge.org/abstract_S0022215100117050

How to cite this article:

R. M. Irving, N. S. Jones, M. A. Hall-Craggs and B. Kendall (1991). CT and MR imaging in lateral sinus thrombosis. The Journal of Laryngology \& Otology, 105, pp 693-695 doi:10.1017/S0022215100117050

Request Permissions : $\underline{\text { Click here }}$ 


\title{
View from Within: Radiology in Focus
}

\section{$\mathrm{CT}$ and MR imaging in lateral sinus thrombosis}

\author{
R. M. Irving, F.R.C.S., N. S. Jones, F.R.C.S., M. A. Hall-Craggs, F.R.C.R.* , B. Kendall, F.R.C.R.* \\ (London)
}

\begin{abstract}
Establishing a pre-operative diagnosis of lateral sinus thrombosis can be difficult, as the clinical features are non-specific and laboratory tests unhelpful. CT may demonstrate abnormal high density of the lumen of the sinus, which does not enhance after intravenous contrast medium. Enhancement of the dura surrounding the sinus may be prominent, causing the 'empty triangle' or 'delta sign' which may suggest the diagnosis. Magnetic resonance (MR) imaging may show both lack of flow and abnormal signal from the sinus, thus providing definitive evidence of thrombosis.
\end{abstract}

\section{Introduction}

Intracranial complications secondary to otological disease remain life threatening conditions, early recognition and treatment being essential for success. Lateral sinus thrombosis presents with few localising features rendering prompt diagnosis difficult.

Spread of infection from the middle ear or mastoid, whether direct or via emissary veins, can result in a perisinus abscess, giving rise to headache, malaise and the characteristic "picket fence' fever curve. A progressive thrombophlebitis can
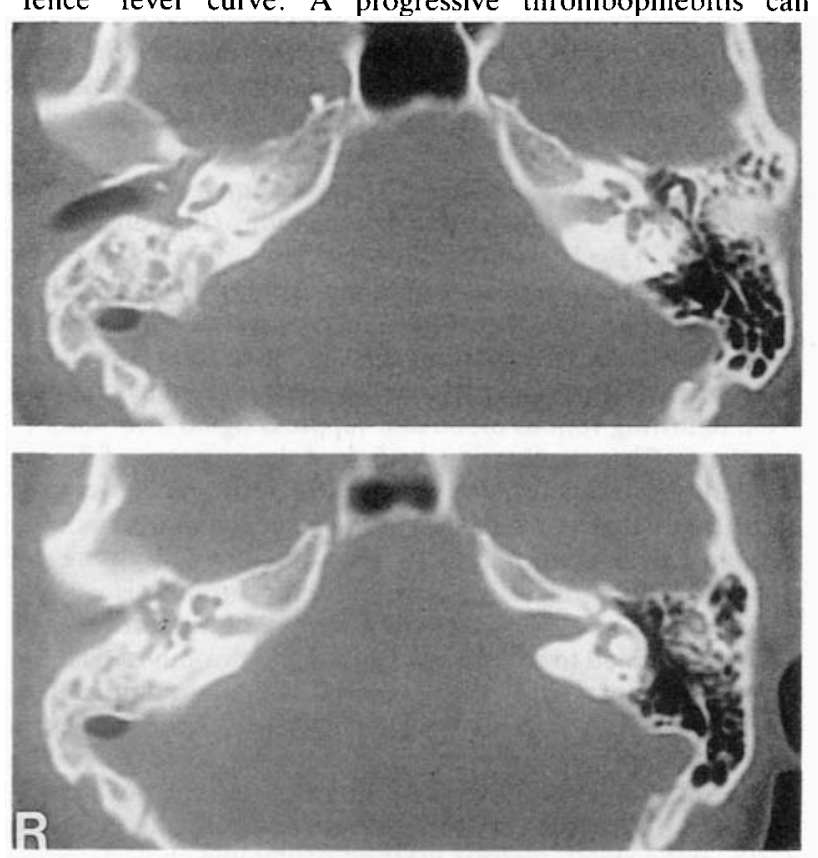

FIG. 1

High resolution computed tomogram contiguous sections made through temporal bones with wide window. The right middle ear and mastoid air cells are opaque. There is erosion of bone around the mastoid antrum and of the auditory ossicles. Gas in the right sinodural angle indicates either an abscess caused by a gas forming organism or a fistula due to erosion into the angle from the mastoid. develop with the potential for both local and distant (embolic) sequale. Mastoid emissary vein thrombosis leads to pitting oedema over the occiput (Griesinger's sign). Neck and mastoid tenderness, as well as torticollis and neck abscesses, have also been reported. Metastatic disease caused by infected thrombi may occur with consequent pneumonia, septic infarcts, osteomyelitis or renal abscess. Occasionally impaired venous drainage and cerebrospinal fluid reabsorption ensue, producing a rise in intracranial pressure with hydrocephalus (otitic), papilloedema, seizures and, rarely, coma.

Investigations other than CT and MR imaging are of limited help; a neutrophil leucocytosis of greater than 20,000 is suggestive, as is a progressive anaemia. A lumbar puncture should not be performed because of the risk of brain herniation; the Tobey-Ayer test is unreliable, and of historical interest only (Seid and Sellars, 1973; Albert and Williams, 1986; Samuel and Fernandez, 1987). Cerebral arteriography and retrograde jugulography are both invasive procedures; the former may precipitate a stroke and the latter may result in dislodgement of thrombus. Intravenous digital subtraction angiography is less invasive and may produce diagnostic images in co-operative patients.

\section{Case Report}

A 15-year-old girl presented with a week's history of rightsided otorrhoea, followed by headache, vomiting, diplopia, and lower limb weakness. On admission she was fully orientated, with a pyrexia of $38^{\circ} \mathrm{C}$, a left abducens nerve palsy, neck stiffness, a bilateral extensor plantar response, and bilateral papilloedema. Pus was observed arising from an attic defect in the right tympanic membrane, and tenderness was elicited over McEwan's triangle. Her white count was $22.8 \times 10_{9} / \mathrm{L}$., and ESR $68 \mathrm{~mm} / \mathrm{hr}$. She was commenced on parenteral chloramphenicol, metronidazole and benzylpenicillin.

Contrast enhanced CT (Fig. 1) demonstrated poor development of mastoid air cells on the right side, with complete opacification of the right middle ear cavity and mastoid. There was erosion of the malleus and incus suggesting chronic infection with cholesteatoma formation. A loculus of gas was present in the sino-dural angle, suggesting a bone defect between the mastoid and posterior fossa and/or an epidural abscess 

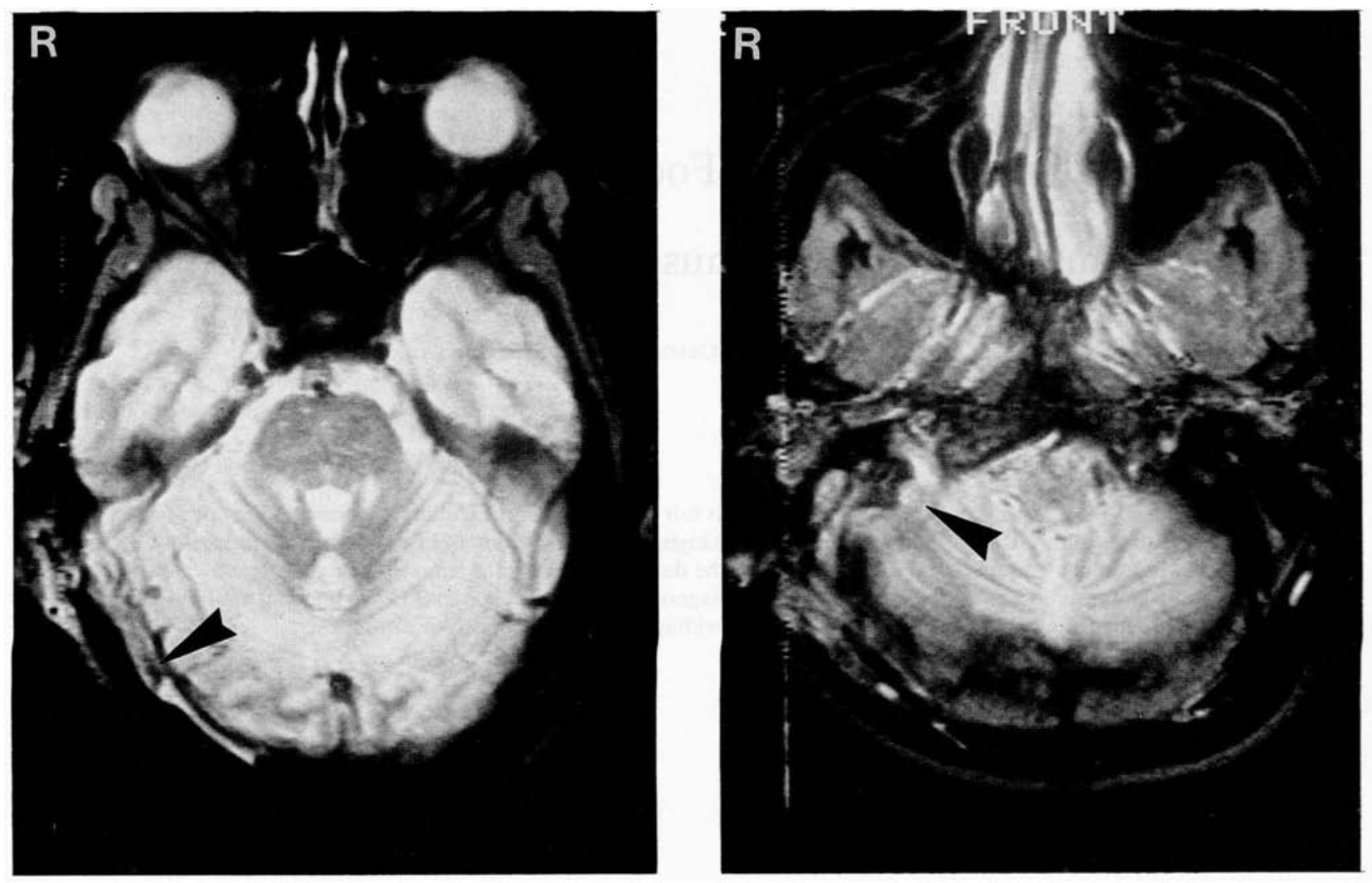

FIGS. $2 a$ and $b$

Transverse STIR (TI $=145 \mathrm{~ms}, T R=3000 \mathrm{~ms}, T E=85 \mathrm{~ms})$ magnetic resonance images at the level of (a) the transverse venous sinus and (b) the sigmoid venous sinus and the jugular foramen. Abnormal signal (arrowed) is present in the right-sided venous sinuses (compare with the flow void in the left venous sinuses).

There was no mass effect, abnormal enhancement, or other confirmatory sign of an abscess. A cranial MR performed after surgical exploration and evacuation of the abscess) showed high signal in the right transverse (Fig. 2a), sigmoid sinuses,

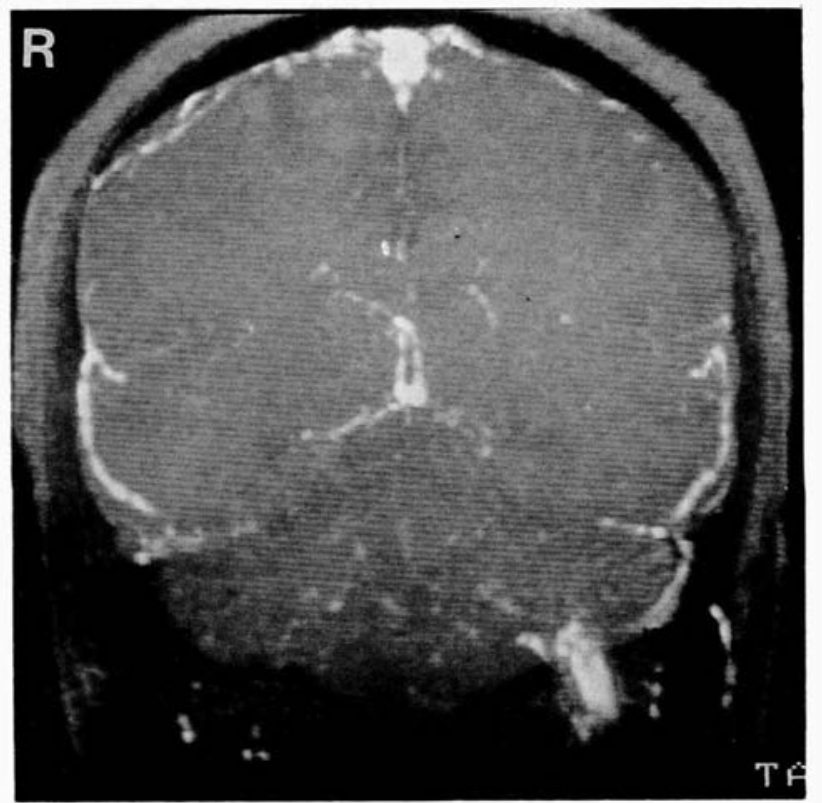

FIG. 3

Maximum intensity projection image of MR venogram (flip angle $40^{\circ}, \mathrm{TR}=40 \mathrm{~ms}, \mathrm{TE}=12 \mathrm{~ms}$ ) showing flow (high signal) in the left sigmoid sinus and proximal jugular vein. No corresponding flow is seen on the right. A combination of abnormal signal with absence of flow is highly suggestive of venous sinus occlusion by thrombus. and upper part of the jugular vein (Fig. 2b), on both the short and long echo of a Short Tau Inversion Recovery STIR, T1/ TR/TE 145/3000/23,85ms) sequence. An MR venogram was performed by acquiring multiple contiguous slices through the sigmoid sinus in a coronal plane using a 2-dimensional gradient echo sequence with flow compensation (flip angle $40^{\circ}$, $\mathrm{TR}=40 \mathrm{~ms}, \mathrm{TE}=12 \mathrm{~ms}$, slice thickness $5 \mathrm{~mm}$, matrix $256 \times 64$ ). A dephasing radiofrequency pulse was applied to the vessels in the neck to eliminate signal from inflowing (arterial) blood. A maximum intensity projection through these images is shown in Figure 3. The purpose of this sequence is to enhance signal from inflowing venous blood and suppress signal from stationary tissue. The examination confirmed the presence of flow in the left sigmoid sinus, the left jugular vein and the sagittal sinus. No signal was detected in the right sigmoid sinus. The combination of abnormal signal from the sinus with absence of flow was highly suggestive of lateral sinus thrombosis.

At surgery, air was noted to 'bubble out' of the cortical bone as it was thinned to expose a collapsed and thrombosed lateral . sinus. The lateral sinus was exposed to the mastoid tip, and to the horizontal sinus where it remained thrombosed. The lateral sinus and dura were covered by a thick yellow exudate, and on retracting these posteriorly, an extradural collection of pus was released. There was no bony erosion of the tegmen or sinus plate, and a small sclerotic mastoid containing cholesteatoma was found. The body of the incus and head of the malleus were eroded. A modified radical mastoidectomy was performed. The internal jugular vein was not ligated, as there were no symptoms or signs of emboli. In order to exclude a coexisting subdural collection of pus, a small incision was made in the dura and clear CSF was obtained. An extradural drain was left in position for 48 hours. Pre-operative blood and operative pus culture both grew Bacillus fragilis and Peptostreptococcus, gasproducing organisms often associated with intracranial suppuration secondary to ear pathology. 
The patient's headache and vomiting resolved within eight hours of surgery, and she remained apyrexial post-operatively. Her abducens nerve palsy resolved after three days. Parentral antibiotics were stopped after two weeks, and oral antibiotics substituted.

\section{Discussion}

The case presented demonstrates many of the typical clinical features of lateral sinus thrombosis with otitic hydrocephalus. The CT scan, while demonstrating valuable information, was non-diagnostic; whereas the MRI was highly suggestive of thrombosis.

Dural sinus thrombosis may be associated with cerebral oedema and multifocal infarction, which is often bilateral and haemorrhagic, and may cause sufficient brain swelling to compress the ventricles. Dilated collateral veins may be visible, and may produce increased enhancement in the tentorium and falx (de Slegte et al., 1988). Uncommonly an abnormally dense sinus on plain CT and a filling defect or 'delta sign' on enhanced CT give more definitive evidence of the diagnosis. In lateral sinus thrombosis, CT is particularly important for demonstration of pathology in the mastoid and adjacent parts of the cranial cavity.

MR scanning may show (as in this case) an abnormal signal from the sinus and lack of flow which, in this clinical situation, is likely to be due to venous sinus occlusion by thrombus. As with any angiographic technique MR venography does not delineate the extent of the thrombus as signal loss distal to the obstruction may be due to (a) higher orders of motion, for example turbulence and acceleration, or (b) saturation of slowly moving blood. However, MR angiography has several advantages; it is a non-invasive technique and does not require the use of intravenous contrast, it can be performed rapidly (in this case in under four minutes) and it does not use ionizing radiation. Gadolinium enhancement of MR images may also show a 'delta sign' comparable to that seen on CT scans. A 'target' pattern of concentric different densities has been reported (Fritsch et al., 1990) and may represent flow within a partially thrombosed sinus.

Prior to the introduction of MRI the diagnosis of otitic hydrocephalus was frequently one of exclusion (SchonstedMadsen et al., 1984); a CT scan confirming normal ventricular size and no space occupying lesion. In a case of suspected sinus thrombosis, without raised intracranial pressure, MRI is also the most likely investigation to be contributory (Hulcelle et al., 1989).

MRI is a non-invasive technique without ionising radiation that accurately delineates the thrombus, affording a positive diagnosis; this is the investigation of choice, and should be performed in conjunction with CT, thereby fully evaluating associated otologic and cerebral pathology.

\section{References}

Albert, D. M., Williams, S. R. (1986) Clinical and anatomical considerations of the Tobey-Ayer test in lateral sinus thrombosis. Journal of Laryngology and Otology, 100: 1311-1313.

de Slegte, R. G. M., Kaiser, M. C., van der Baan, S., Smit, L. (1988) Computed tomographic diagnosis of septic sinus thrombosis and their complications. Neuroradiology, 30: 160-165.

Fritsch, M. H., Miyamoto, R. T., Wood, T. L. (1990) Sigmoid sinus thrombosis diagnosis by contrasted MRI scanning. Otolaryngology Head and Neck Surgery, 103: 451-456.

Hulcelle, P. J., Dooms, G. C., Mathurin, P., Cornelis G. (1989) MRI assessment of unsuspected dural sinus thrombosis. Neu roradiology, 31: 217-221.

Samuel, J. Fernandes, C. M. C. (1987) Lateral sinus thrombosis (A review of 45 cases). Journal of Laryngology and Otology, 101 : 1227-1229.

Seid, A. B., Sellars, S. L. (1973) The management of otologic lateral sinus disease at Groote Schuur Hospital. Laryngoscope, 83: $397-403$.

Schonsted-Madsen, U., Sehested, P., Brask, T. (1984) Benign intracranial hypertension caused by mastoiditis and lateral sinus obstruction: The value of computerised tomography in diagnosis. Journal of Laryngology and Otology, 98: 395-398.

Address for correspondence:

Mr R. M. Irving, F.R.C.S.,

Department of Otolaryngology,

Addenbrookes Hospital,

Hills Road,

Cambridge CB2 2OQ

Key words: Thrombosis; Lateral sinus; Radiography, imaging 